\title{
Mechanism Design Theory: How to Implement Social Goals
}

\author{
Eric Maskin \\ Institute for Advanced Study, Princeton \\ maskin@ias.edu
}

\begin{abstract}
The theory of mechanism design can be thought of as the engineering side of economic theory. One begins by identifying a social or economic goal. The theory then addresses the question of whether or not an appropriate institution or procedure (that is, a mechanism) could be designed to attain that goal.
\end{abstract}

